\title{
Fabrication and Characterization of Finite-Size DNA 2D Ring and 3D Buckyball Structures
}

\author{
Soojin Jo ${ }^{1}\left(\mathbb{D}\right.$, Seungjae Kim ${ }^{2}$, Byung Ho Lee ${ }^{1}$, Anshula Tandon ${ }^{3}$, Byunghoon Kim ${ }^{2}$, \\ Sung Ha Park ${ }^{2,3, *}$ and Moon Ki Kim ${ }^{1,3, *}$ \\ 1 School of Mechanical Engineering, Sungkyunkwan University, Suwon 16419, Korea; jsj89@skku.edu (S.J.); \\ byunghooo@gmail.com (B.H.L.) \\ 2 Department of Physics, Sungkyunkwan University, Suwon 16419, Korea; seungjae@skku.edu (S.K.); \\ werft2@skku.edu (B.K.) \\ 3 Sungkyunkwan Advanced Institute of Nanotechnology (SAINT), Sungkyunkwan University, \\ Suwon 16419, Korea; anshula.tandon@gmail.com \\ * Correspondence: sunghapark@skku.edu (S.H.P.); mkkim@me.skku.ac.kr (M.K.K.); \\ Tel.: +82-31-299-4840 (M.K.K.)
}

Received: 9 May 2018; Accepted: 25 June 2018; Published: 27 June 2018

\begin{abstract}
In order to incorporate functionalization into synthesized DNA nanostructures, enhance their production yield, and utilize them in various applications, it is necessary to study their physical stabilities and dynamic characteristics. Although simulation-based analysis used for DNA nanostructures provides important clues to explain their self-assembly mechanism, structural function, and intrinsic dynamic characteristics, few studies have focused on the simulation of DNA supramolecular structures due to the structural complexity and high computational cost. Here, we demonstrated the feasibility of using normal mode analysis for relatively complex DNA structures with larger molecular weights, i.e., finite-size DNA 2D rings and 3D buckyball structures. The normal mode analysis was carried out using the mass-weighted chemical elastic network model (MWCENM) and the symmetry-constrained elastic network model (SCENM), both of which are precise and efficient modeling methodologies. MWCENM considers both the weight of the nucleotides and the chemical bonds between atoms, and SCENM can obtain mode shapes of a whole structure by using only a repeated unit and its connectivity with neighboring units. Our results show the intrinsic vibrational features of DNA ring structures, which experience inner/outer circle and bridge motions, as well as DNA buckyball structures having overall breathing and local breathing motions. These could be used as the fundamental basis for designing and constructing more complicated DNA nanostructures.
\end{abstract}

Keywords: finite-size; DNA structure; self-assembly; elastic network model; normal mode analysis

\section{Introduction}

DNA has emerged as a fascinating molecule for self-assembly in structural DNA nanotechnology due to its inherent molecular recognition, base-sequence programmability, well-structured conformation, and predictable nanoscale dimensions [1-3]. The essence of this burgeoning field is the construction of a wide variety of novel structures via self-assembly of DNA building blocks on the nanometer scale with high precision. Self-assembly of these nucleic acid building blocks can be used to design and construct various dimensional nanostructures. To date, there have been many experimental attempts to construct various DNA nanostructures including one-dimensional (1D) tubes [4], 2D lattices [5-8], 2 and 3D molecular canvases [9-11], as well as finite-size 2D rings [12,13] and 3D polyhedra [14-17]. 
Simulation-based studies are useful for obtaining clues related to the geometric stabilities, specific functionalities, and dynamic characteristics of DNA nanostructures. Normal mode analysis (NMA) has been widely used to analyze intrinsic modes of biomolecules due to its wide adaptability and relatively low cost. Collective motions of biomolecules have a significant influence on their structural characteristics [18-21]. There have been considerable efforts to analyze vibrational characteristics based on NMA for various DNA/RNA native structures [22-26]. Although dynamic analysis for some fabricated nanostructures has also been performed by NMA [20,21,27-31], finite-size nanostructures that have more complex geometries, as well as larger molecular weights, have rarely been studied.

In this study, we introduced two representative finite-size DNA nanostructures, i.e., 2D rings and 3D buckyballs, and performed computational analysis on them using NMA based on a mass-weighted chemical elastic network model (MWCENM) and a symmetry-constrained elastic network model (SCENM) [21,32]. Intrinsic vibrations of the DNA 2D ring and 3D buckyball structures can be determined precisely and efficiently because MWCENM considers both molecular weights and chemical bonds, and SCENM uses the information of a repeated unit and connectivity between adjacent units.

\section{Results}

\subsection{DNA 2D Ring and 3D Buckyball Synthesis Results}

Finite-size DNA 2D ring structures, i.e., open- and closed-formed configurations, were fabricated as shown in Figure 1 . An open-formed ring (referred to as the $\mathrm{R} 1_{\mathrm{O}}$ ring) is made of 12 units of $\mathrm{R} 1_{\mathrm{O}}$ tiles. Each tile contains an eight-nucleotide long single-stranded domain that provides the flexibility of a duplex. In contrast, a closed-formed ring (referred to as the $\mathrm{R} 1_{\mathrm{C}}$ ring) does not have a single-stranded domain. Each $\mathrm{R} 1_{\mathrm{O}}$ and $\mathrm{R} 1_{\mathrm{C}}$ tile has two sticky end pairs (known as the complementary sticky ends shown as a\# and a\#' in Figure 1a,b) for hybridization. Both rings have outer and inner diameters of $29 \mathrm{~nm}$ and $13 \mathrm{~nm}$, respectively. DNA base sequences of $R 1_{O}$ (indicated in blue) and $R 1_{C}(r e d)$ rings and the corresponding atomic force microscope (AFM) images (dimensions are in good agreement with the design) are shown in Figure 1a,b. In addition, $\mathrm{R} 2$ rings ( $\mathrm{R} 2_{\mathrm{O}}$ or $\mathrm{R} 2_{\mathrm{C}}$ rings) containing connection tiles (referred to as $\mathrm{R} 2_{\mathrm{Con}}$ tiles), which make a ring relatively larger than $\mathrm{R} 1$ rings, were constructed in order to demonstrate the feasibility of designing various DNA ring diameters and study size-dependent vibrational characteristics. A representative AFM image of $\mathrm{R} 2 \mathrm{C}$ rings, which agreed with the designed dimensions (the outer and inner diameters of DNA rings are $55 \mathrm{~nm}$ and $40 \mathrm{~nm}$, respectively), is shown in Figure 1c-f.

Finite-size DNA 3D buckyball structures that consisted of 60 three-point-star (3PS) building blocks (consisting of seven individual strands with threefold rotational symmetry) were constructed as shown in Figure 2. The buckyball represents a cage-like structure composed of 20 hexagons and 12 pentagons containing 60 vertexes, 90 edges, and 32 faces. In a 3PS tile, a long central strand with three single-stranded T-loops (provided spatial flexibility) and single-stranded overhangs (that serve as sticky ends) allowed the formation of a 3D geometry, i.e., a DNA buckyball. AFM and transmission electron microscope (TEM) images of the DNA 3D buckyball structure are shown in Figure 2c. Although the buckyball structures shown in the AFM image were not guaranteed to possess 60 3PS tiles, the diameter of the structure $(\sim 80 \mathrm{~nm})$ roughly agreed with the designed scheme. 


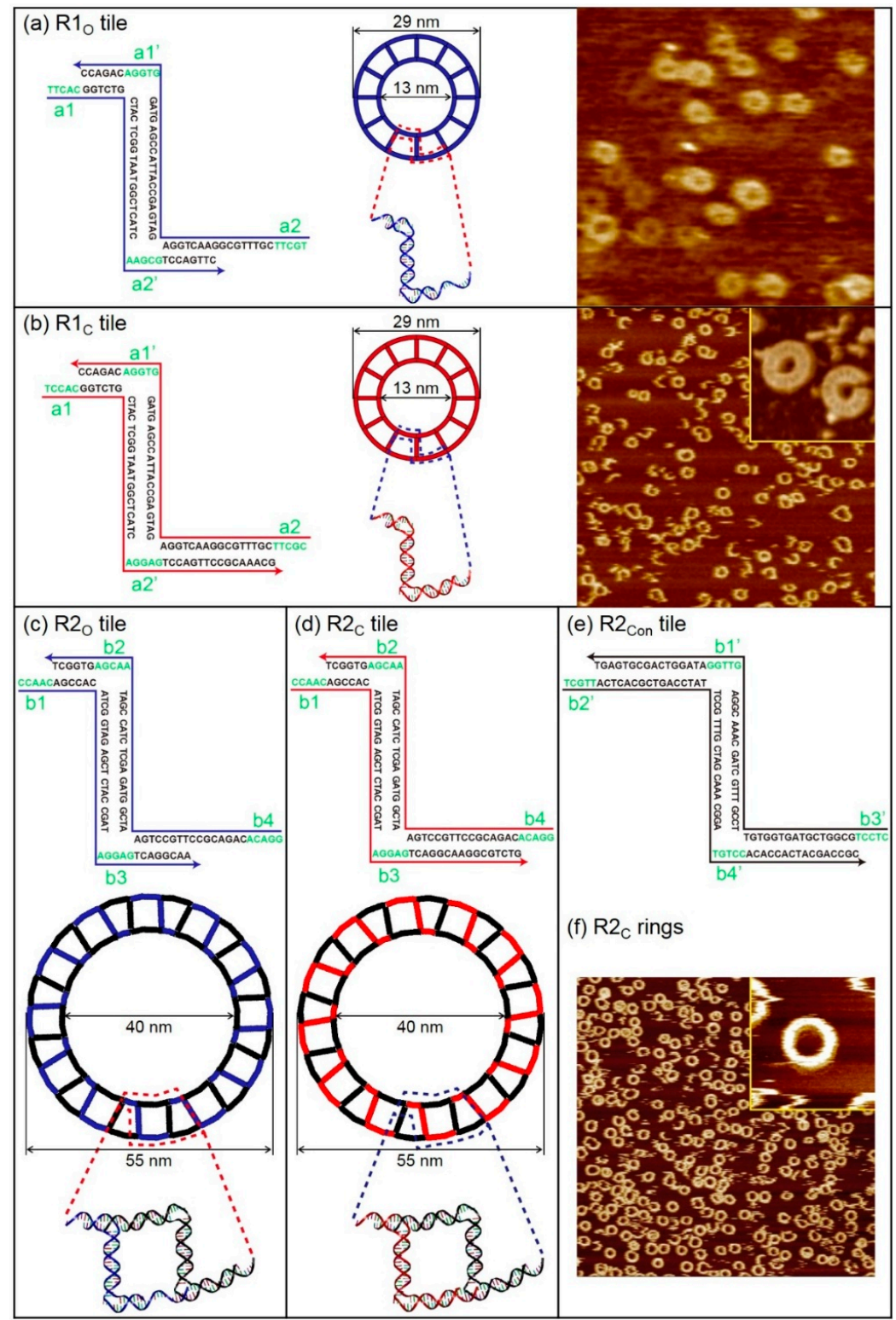

Figure 1. Schematics of finite-size DNA 2D ring structures with their unit tile sequences and their representative atomic force microscope (AFM) images. (a) DNA base sequence of the open-formed unit building block (referred to as an $\mathrm{R} 1_{\mathrm{O}}$ tile), schematic diagram, and corresponding AFM images of DNA 2D ring structures composed of $\mathrm{R} 1_{\mathrm{O}}$ tiles (referred to as an $\mathrm{R} 1_{\mathrm{O}}$ ring) indicated in blue. The complementary sticky end pairs are shown as a\# and a\#'. The outer and inner diameters of DNA rings are $29 \mathrm{~nm}$ and $13 \mathrm{~nm}$, respectively. Here, the scan size of the image is $500 \times 500 \mathrm{~nm}^{2}$. (b) DNA base sequence of the closed-formed unit building block (referred to as an $\mathrm{R} 1_{\mathrm{C}}$ tile), schematic diagram, and corresponding AFM images of DNA 2D ring structures composed of $\mathrm{R} 1_{\mathrm{C}}$ tiles (referred to as an $\mathrm{R} 1_{C}$ ring) indicated in red. Scan sizes of the image and the magnified image in the inset are $1 \times 1 \mu \mathrm{m}^{2}$ and $80 \times 80 \mathrm{~nm}^{2}$, respectively. (c-e) DNA base sequences of open-formed (closed-formed) and connection unit building blocks (referred to as $\mathrm{R}_{2} \mathrm{O}\left(\mathrm{R} 2_{\mathrm{C}}\right)$ and $\mathrm{R} 2_{\mathrm{Con}}$ tiles, respectively) and schematic diagram of the DNA $2 \mathrm{D}$ ring structure composed of $\mathrm{R} 2_{\mathrm{O}}\left(\mathrm{R} \mathrm{C}_{\mathrm{C}}\right)$ and $\mathrm{R} 2_{\mathrm{Con}}$ tiles (referred to as an $\mathrm{R} 2 \mathrm{O}\left(\mathrm{R} 2_{\mathrm{C}}\right)$ ring) indicated in blue (red) and black, respectively. The complementary sticky end pairs are shown as b\# and b\#'. The outer and inner diameters of the DNA rings are $55 \mathrm{~nm}$ and $40 \mathrm{~nm}$, respectively. (f) Representative AFM image of $\mathrm{R} 2_{\mathrm{C}}$ rings. Scan sizes of the image and the magnified image in the inset are $2 \times 2 \mu \mathrm{m}^{2}$ and $100 \times 100 \mathrm{~nm}^{2}$, respectively. 


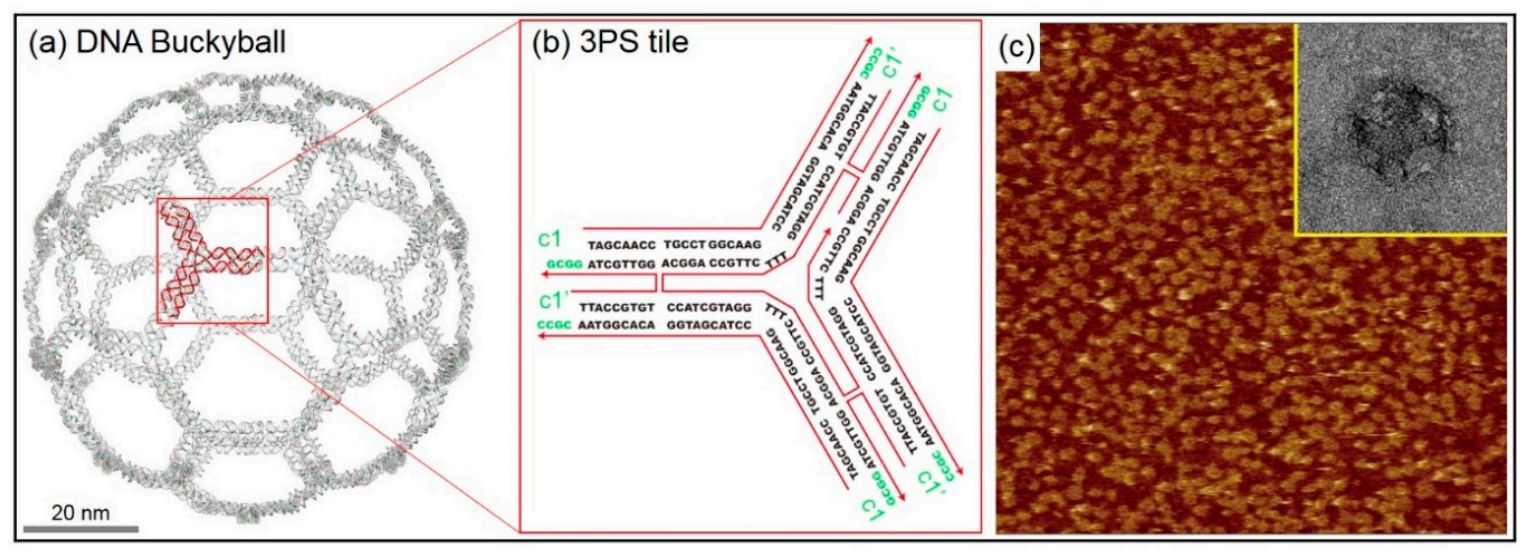

Figure 2. Finite-size DNA 3D buckyball structure with its unit tile sequence, representative atomic force microscope (AFM) and transmission electron microscope (TEM) images. (a) Schematic diagram of a DNA 3D buckyball structure composed of three-point-star (3PS) unit tiles. (b) DNA base sequence of a 3PS unit tile. The complementary sticky end pairs are shown as $\mathrm{c} 1$ and $\mathrm{c1} 1^{\prime}$. (c) AFM and TEM images of the DNA 3D buckyball structure. Scan sizes of the AFM and the TEM images in the inset are $3 \times 3 \mu \mathrm{m}^{2}$ and $100 \times 100 \mathrm{~nm}^{2}$, respectively.

\subsection{Normal Mode Analysis Results of DNA 2D Ring and 3D Buckyball Structures}

Three-dimensional computer models were needed to analyze vibrational characteristics of DNA $2 \mathrm{D}$ ring and 3D buckyball structures. For 2D rings, we can construct their structures by using only their unit tile coordinates with 12 rotation matrices because they have 12-fold ring structures. After modeling different unit tiles of $\mathrm{R} 1_{\mathrm{O}}, \mathrm{R} 1_{\mathrm{C}}, \mathrm{R} 2_{\mathrm{O}}$, and $\mathrm{R} 2_{\mathrm{C}}$ rings on the $\mathrm{xy}$-plane based on their base sequences, their entire ring structures were constructed by rotating every $30^{\circ}$ about the $z$-axis. Likewise, we can construct the 3D buckyball structure by using its unit tile coordinates with 60 rotation matrices because it is an icosahedron symmetry structure with 60 repeated 3PS unit tiles. First, the 3PS unit tile was generated on the xy-plane based on its base sequence. The whole buckyball structure was constructed using the 3D coordinates of the unit tile and 60 rotation matrices referenced from the HK97 virus structure, which also consisted of 60 repeated units [33]. In order to verify appropriate assignment between adjacent unit tiles in the constructed buckyball, the number of hydrogen bonds at their sticky ends was evaluated. After modeling finite-size 2D rings and 3D buckyball, we confirmed their structural reliabilities by calculating both the hydrogen bond length between complementary base pairs and distances between two adjacent atoms. Appropriate binding between unit tiles was verified in all 3D computer models and no steric clashing was found.

Both MWCENM and SCENM were then applied to these 3D computer models to obtain their intrinsic vibration modes. Figure 3 shows the major vibrational modes of 2D rings. Their dominant modes are classified into three different motions, including the outer circle motion (i.e., zigzag motion), inner circle motion (i.e., out-of-plane translation, in-plane translation, and out-of-plane rotation of the inner circle), and bridge motion (i.e., spiderlike and mixed spiderlike motions). The first three lowest modes show inner circle motions for all 2D rings, such as out-of-plane translation of the inner circle at the 1st mode and out-of-plane rotation of the inner circle at both the 2 nd and the 3 rd modes. At the 4th mode, spiderlike motion and zigzag motion were present at the $\mathrm{R} 1$ rings ( $\mathrm{R} 1_{\mathrm{O}}$ and $\mathrm{R} 1_{\mathrm{C}}$ rings) and $\mathrm{R} 2$ rings ( $\mathrm{R} 2 \mathrm{O}$ and $\mathrm{R} 2_{\mathrm{C}}$ rings), respectively. Bridge motions appeared more frequently in $\mathrm{R} 1$ rings, but $\mathrm{R} 2$ rings had no bridge motion below the 8 th lowest mode. Also, the outer circle motions appeared more frequently in $\mathrm{R} 2$ rings compared to R1 rings. In-plane translation of the inner circle was shown only at R1 rings higher than the 7th mode. Over the 9th mode, the mixed outer circle and bridge motions or outer circle and inner circle motions were observed in all 2D rings. Therefore, DNA 2D rings showed six different major vibrational modes. Overall, their vibrational characteristics depended on their size, and there was no significant difference between the open-formed and closed-formed configurations. 


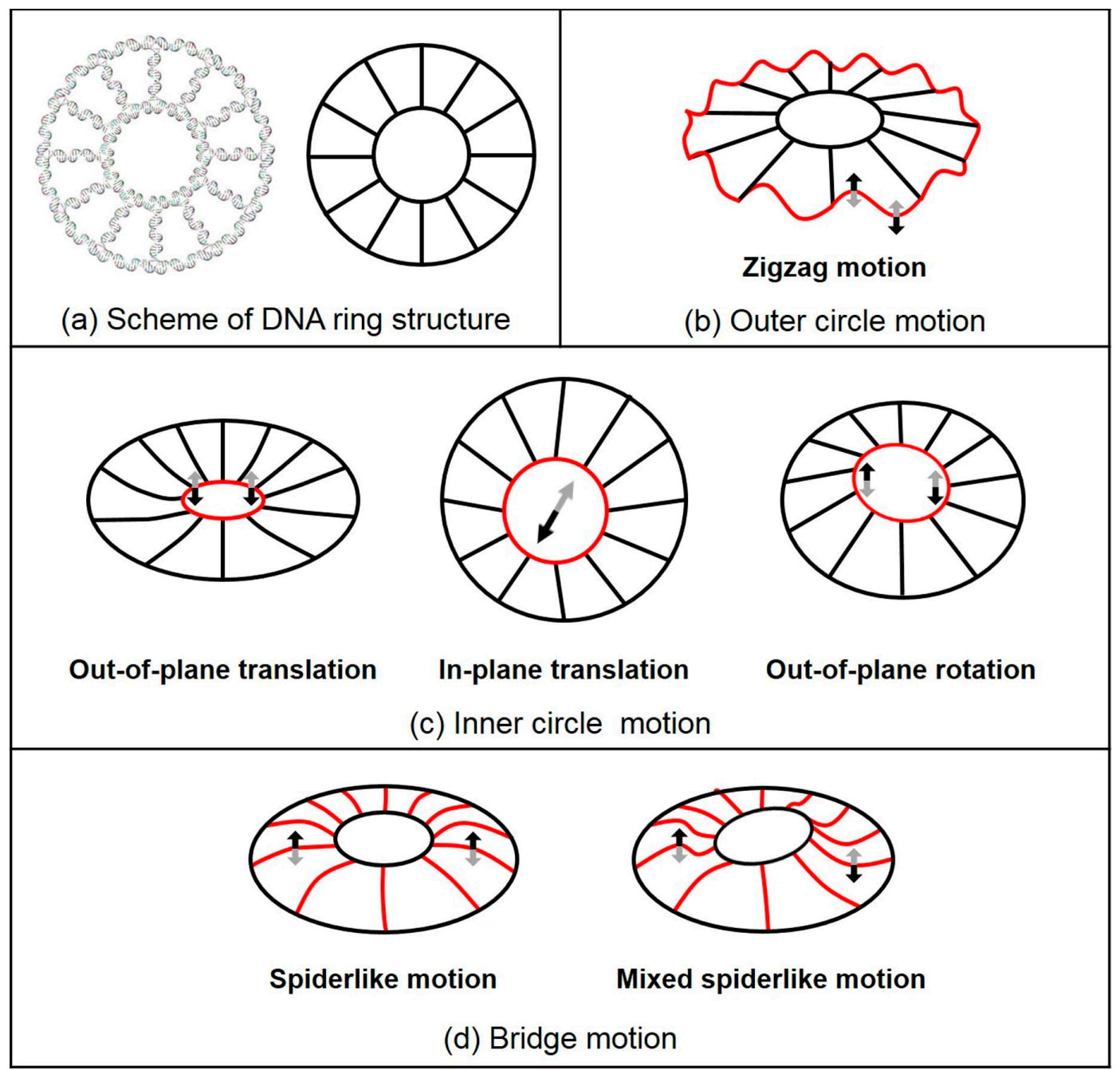

Figure 3. Schematics of the DNA 2D ring structure and its major vibration modes. These modes are classified into three different motions, including outer circle motion, inner circle motion, and bridge motion. (a) Scheme of the DNA ring structure. (b) Zigzag motion, outer circle motion, is sinusoidal and the shape of outer circle is red. The 8th-10th mode at R1 rings and the 4th-10th mode at R2 rings are shown. (c) Inner circle motions consist of out-of-plane translation, in-plane translation, and out-of-plane rotation of inner circle (indicated in red). Out-of-plane and in-plane translations are the inner circle of the DNA ring structure moving in vertical and lateral directions, respectively. Out-of-plane rotation is the seesaw motion of the inner circle. For all ring structures, out-of-plane translation and out-of-plane rotation are dominant motions, as shown in the 1st mode and both the 2nd and 3rd modes, respectively. In-plane translation can be observed only at R1 rings over the 7th mode. (d) Bridge motion is composed of spiderlike and mixed spiderlike motions. Spiderlike motion is the bridge bending motion in the upper direction and is observed in all ring structures. It is present in the 4 th mode of $\mathrm{R} 1$ rings. Mixed spiderlike motion is also shown in both the 5 th and the 6 th modes of the R1 rings.

In addition, we calculated normal modes for both the buckyball and its 3PS unit tile structures. Vibrational mode shapes of the unit tile structure can be easily obtained by using MWCENM-based NMA. In the case of the 3D buckyball structure, however, we additionally adopted the SCENM method based on its icosahedral symmetry in order to reduce the computational cost in NMA due to its large structural size (i.e., the number of coarse-grained atoms is 102,960 ). Finally, we successfully obtained mode shapes of the 3D buckyball structure by using only the unit tile coordinates along with its information related to connectivity with neighboring tiles. These results are 
shown in Figures 4 and 5, respectively. Figure 4 shows the major vibrational modes of the 3PS unit tile. It primarily shows bending motion, tweezer-like motion, and twisting or bending at the sticky ends. The bending motion is on the 1st lowest mode, and tweezer-like motion appeared at the 3rd and the 8th modes. Other modes below the 10th lowest mode are bending, twisting, and mixed motion of bending and twisting at sticky ends. Based on these dominant modes of the unit tile, we found that 3PS unit tiles can be assembled as spherical buckyball structures. Figure 5 shows the dominant vibrational modes of the whole buckyball structure. These major modes are overall breathing and local breathing motions. The overall breathing motion appeared at the 1st and the 2nd modes, and the local breathing motion was observed from the 4th-7th modes. Over the 8th mode, the mixed local breathing and other motions were observed. Uncommon torsional motion appeared at the 3rd mode. In this mode, the motion of the unit tile is composed of two stationary sticky ends and one twisting sticky end. The first 10 lowest modes for all finite-size DNA rings and buckyball structures are summarized in Table 1.

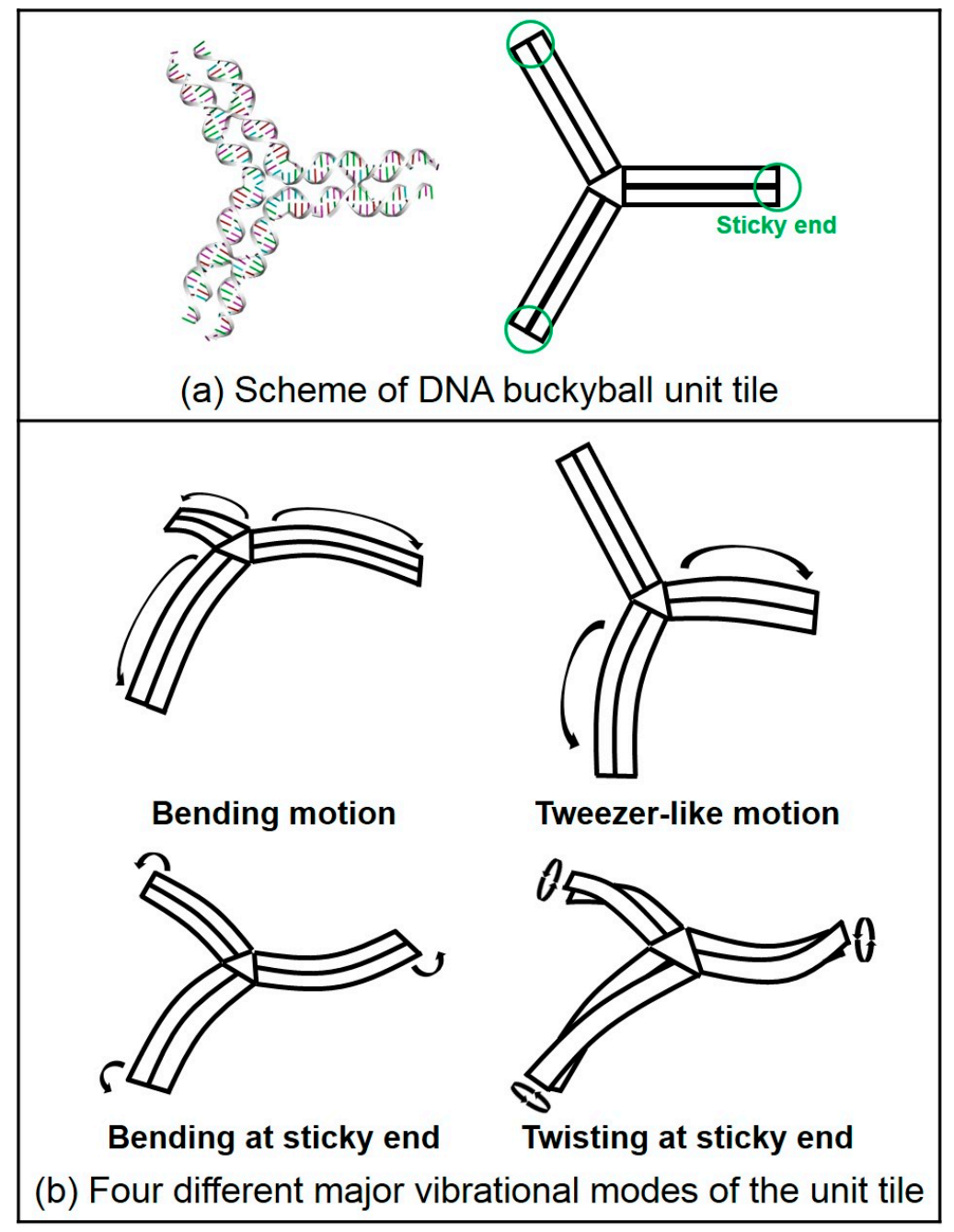

Figure 4. Schematics of three-point-star (3PS) unit tile and its major vibration modes. (a) Scheme of 3PS unit tile. DNA duplex is represented as a rectangle, and its three sticky ends are marked with green circles. (b) The unit tile shows four different major vibrational modes such as bending motion, tweezer-like motion, and bending or twisting at sticky ends. Within the 10th lowest mode, the bending motion is the 1st mode and both the 3rd and the 8th modes are tweezer-like motions. Other modes show bending, twisting or mixed bending and twisting at sticky ends. 


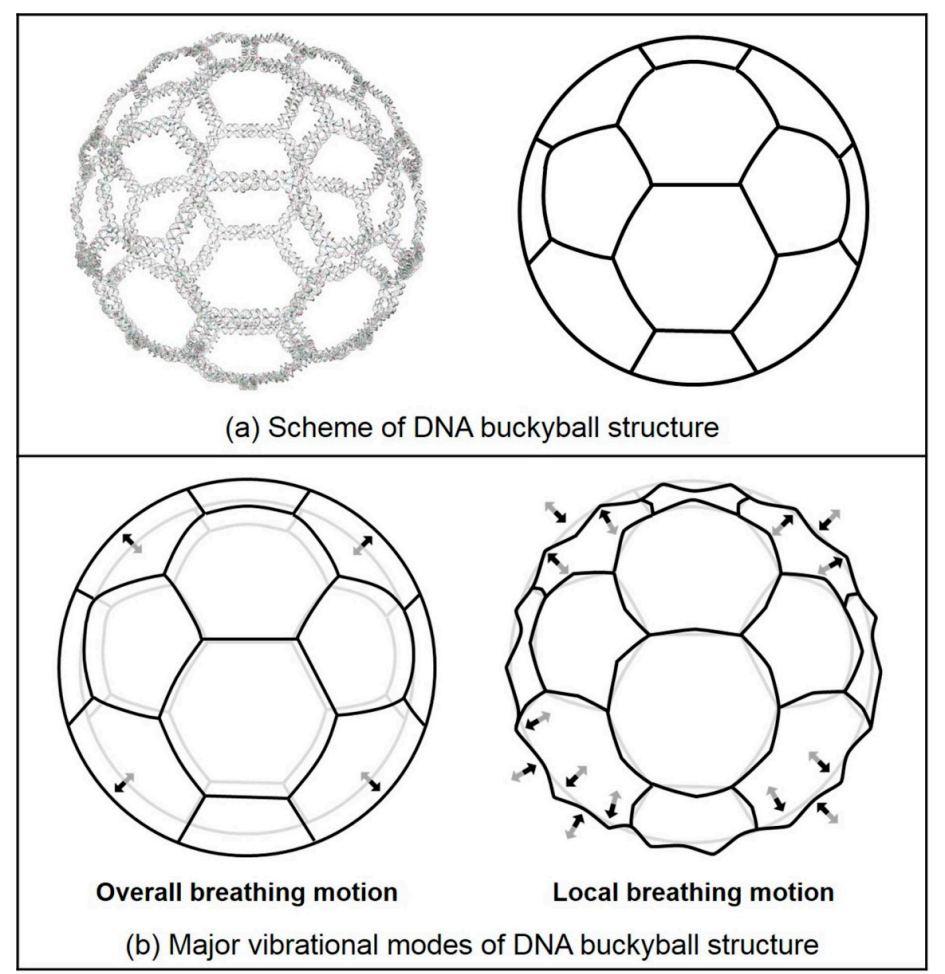

Figure 5. Schematics of the DNA buckyball structure and its major vibration modes. (a) The scheme of the DNA buckyball is represented as soccer ball. (b) Major vibrational modes of the DNA buckyball are overall breathing motion and local breathing motion. Overall breathing motion is the swelling of the entire buckyball structure, and local breathing motion forms a spiky shape. Its conformational change from initial to final structures is depicted in gray and black, respectively.

Table 1. Vibrational mode shapes of finite-size DNA 2D ring and 3D buckyball structures.

\begin{tabular}{|c|c|c|c|c|c|}
\hline \multirow{2}{*}{ Mode Number } & \multicolumn{5}{|c|}{ Mode Shape } \\
\hline & $\mathbf{R} 1_{\mathbf{o}}$ Ring & R1 $1_{c}$ Ring & R2 ${ }_{0}$ Ring & R2 $2_{c}$ Ring & Buckyball \\
\hline Mode 1 & $\mathrm{O}-\mathrm{T}^{\mathrm{a}}$ motion & O-T motion & O-T motion & O-T motion & $\mathrm{O}-\mathrm{B}^{\mathrm{e}}$ motion \\
\hline Mode 2 & $\mathrm{O}-\mathrm{R}^{\mathrm{b}}$ motion & O-R motion & O-R motion & O-R motion & O-B motion \\
\hline Mode 3 & O-R motion & O-R motion & O-R motion & O-R motion & Torsional motion \\
\hline Mode 4 & Spiderlike motion & Spiderlike motion & Zigzag motion & Zigzag motion & L-B ${ }^{\mathrm{f}}$ motion \\
\hline Mode 5 & M-S ${ }^{\mathrm{c}}$ motion & M-S motion & Zigzag motion & Zigzag motion & L-B motion \\
\hline Mode 6 & M-S motion & M-S motion & Zigzag motion & Zigzag motion & L-B motion \\
\hline Mode 7 & Spiderlike motion & $\mathrm{I}^{\mathrm{T}}{ }^{\mathrm{d}}$ motion & Zigzag motion & Zigzag motion & L-B motion \\
\hline Mode 8 & Mixed motion & I-T motion & Zigzag motion & Zigzag motion & Mixed motion \\
\hline Mode 9 & Mixed motion & Mixed motion & Mixed motion & Zigzag motion & Mixed motion \\
\hline Mode 10 & Mixed motion & Mixed motion & Zigzag motion & Mixed motion & Mixed motion \\
\hline
\end{tabular}

a O-T: Out-of-plane translation; ${ }^{\mathrm{b}}$ O-R: Out-of-plane rotation; ${ }^{\mathrm{c}} \mathrm{M}-\mathrm{S}$ : Mixed spiderlike; ${ }^{\mathrm{d}}$ I-T: In-plane translation;

e O-B: Overall breathing; ${ }^{\mathrm{f}}$ L-B: Local breathing.

\section{Discussion}

In summary, we designed, fabricated, and characterized finite-size DNA 2D ring and 3D buckyball structures. DNA 2D ring and 3D buckyball structures were successfully fabricated, and their physical geometries were verified through AFM and TEM. In addition, 3D computer models were constructed for these structures, which were then used to characterize their vibrational features based on both MWCENM- and SCENM-based NMA. When establishing 3D computer models, their structural reliability was confirmed by evaluating the number of hydrogen bonds and distances between adjacent atoms. The $2 \mathrm{D}$ ring results of NMA showed that major vibration modes were categorized into three different motions, including inner circle motion, outer circle motion, and bridge motion. Although size-dependent vibrational properties were observed, there was no significant configuration 
difference between open- and closed-formed unit tiles. Both the unit tile and whole structures were analyzed for a 3D buckyball. Dominant vibration motions of the unit tile include bending, tweezer-like motion, and twisting or bending at the sticky ends. These modes seem to cooperate with each other to assemble spherical buckyball structures from unit tiles. Overall breathing and local breathing motions were mainly observed for the whole structure. In addition, the proposed NMA methodology is expected to play an important role in the construction of a structural and functional data library of various DNA nanostructures, as well as other macromolecules. This data library will lead to fundamental advances in simulation-based design, fabrication, and functionalization of DNA nanostructures by helping to understand their assembly mechanism and dynamics.

\section{Materials and Methods}

\subsection{Synthesis Method}

High-performance liquid chromatography purified synthetic oligonucleotides were purchased from BIONEER (Daejeon, Korea). DNA samples, i.e., DNA 2D ring and 3D buckyball structures, were formed by mixing a stoichiometric quantity of each strand in a buffer, $1 \times \mathrm{TAE} / \mathrm{Mg}^{2+}(40 \mathrm{mM}$ Tris base, $20 \mathrm{mM}$ acetic acid, $1 \mathrm{mM}$ EDTA ( $\mathrm{pH} 8.0$ ), and $12.5 \mathrm{mM}$ magnesium acetate). Final concentrations of $100 \mathrm{nM}$ (for DNA 2D rings) and $500 \mathrm{nM}$ (for 3D buckyball structures) were achieved. For annealing, the DNA strands were added to a test-tube with a total sample volume of $100 \mu \mathrm{L}$ and were then placed in a Styrofoam box with $2 \mathrm{~L}$ of boiled water to cool slowly from $95^{\circ} \mathrm{C}$ to $25^{\circ} \mathrm{C}$ over a period of at least $24 \mathrm{~h}$ to facilitate hybridization.

\subsection{AFM and TEM Measurements}

For AFM imaging, the DNA sample was placed on a metal puck using instant glue. Then, $20 \mu \mathrm{L}$ of $1 \times \mathrm{TAE} / \mathrm{Mg}^{2+}$ buffer was pipetted onto the sample substrate, and another $20 \mu \mathrm{L}$ of $1 \times \mathrm{TAE} / \mathrm{Mg}^{2+}$ buffer was dispensed onto a silicon nitride AFM tip (Veeco Inc., San Jose, CA, USA). The AFM images were obtained by using a Multimode Nanoscope (Veeco Inc., USA) in liquid tapping mode. Transmission electron micrographs were obtained on a JEOL JEM-ARM300F for TEM imaging. Direct measurement of DNA 3D buckyball structures was carried out by pipetting $5 \mu \mathrm{L}$ of an annealed DNA solution onto a carbon film-layered copper grid. Negative staining involved exposure of the sample on the grid to $10 \mu \mathrm{L}$ of $2 \%$ uranyl acetate solution for a few minutes before imaging.

\subsection{Mass-Weighted Chemical Elastic Network Model (MWCENM)}

In an elastic network model (ENM), representative atoms are modeled as unit point masses, and connectivity between atoms is represented as a unit spring constant that varies depending on the cutoff distance, which is usually $12 \AA$ in a $C_{\alpha}$ coarse-grained protein model [34-38]. ENM methodology has been mainly used to analyze intrinsic mode shapes of proteins, but recently, it has also been used to analyze intrinsic vibrational features of DNA/RNA structures because of its advantages (i.e., low computational cost and large conformational change prediction) compared to the traditional full-atom molecular dynamics (MD) simulations [20-30]. In comparison with a traditional ENM, the MWCENM is a more precise modeling method because it considers both the inertia effect and the chemical bond information of the target system. The inertia effect is given by lumped masses to representative atoms of the target system, and chemical bonds are considered by assigning different spring constants, as shown in Table $2[19,21]$. Here, we assigned a lumped mass of the surrounding atoms to each representative atom. Each nucleotide is represented by six or seven atoms. Three atoms (P, C4, and C1) represent the sugar-phosphate backbone. In the pyrimidine (purine) base, three (four) atoms related to the connection with backbone or hydrogen bonds between complementary bases are selected as representative atoms [21]. A spring network of a coarse-graining model is constructed depending on the type of chemical bonds. Van der Waals interactions, hydrogen bonds, and covalent 
bonds are determined based on a cut-off distance of $8 \AA$, complementary base information, and DNA linkages, respectively, and their spring constant ratios are 1:10:100. Therefore, MWCENM enables us to calculate both the frequency values and corresponding mode shapes of the target system.

Table 2. Various spring constants assigned to each type of chemical bond in MWCENM.

\begin{tabular}{cc}
\hline Chemical Bond & Spring Constant (N/m) \\
\hline Van der Waals & 7 \\
Hydrogen bond & 70 \\
Ion bond & 70 \\
Disulfide bond & 700 \\
Covalent bond & 700 \\
\hline
\end{tabular}

\subsection{Symmetry-Constrained Elastic Network Model (SCENM)}

If the target system has a symmetric structure composed of repeated subunit structures, we can use symmetry-constrained ENM (SCENM) to analyze full models effectively by using only a repeated subunit structure with connectivity information of neighboring subunits instead of handling all the structural information $[32,39,40]$. The advantage of SCENM is its ability to reflect the crystal packing effect of protein structures with reduced computational cost [41]. The computational cost is reduced as $1 / N$ if the target structure consists of $N$ copies of its subunit structure.

\subsection{Normal Mode Analysis (NMA)}

We performed MWCENM-based NMA to analyze vibrational characteristics of four different DNA 2D rings and the 3PS unit tile of a 3D buckyball. Suppose there is a system of $N$ particles. Each particle is a representative atom chosen through sampling and a lumped mass that includes the weight of surrounding atoms. We constructed a spring network between particles using the chemical bond types (Table 2) and then constructed the equation of motion (EOM) for the system as follows. The position of the $i$ th atom at time $t x_{i}(t)$ can be represented by the initial position $x_{i}(0)$ and a small displacement $\delta(t)$ so that $x_{i}(t)=x_{i}(0)+\delta(t)$, where $x_{i}(t)$ is a $3 \times 1$ vector. Then, the kinetic energy of the system can be defined as

$$
T=\frac{1}{2} \sum_{i=1}^{n} m_{i}\left\|\dot{x}_{i}(t)\right\|^{2},
$$

where $m_{i}$ is the weight of a specific lumped mass. The kinetic energy can be rewritten in matrix form as

$$
T=\frac{1}{2} \dot{\delta}^{T} M \dot{\delta}
$$

where $M$ is a global mass matrix consisting of the lumped masses and $\delta$ is a $3 N \times 1$ matrix. In addition, the potential energy can be written as

$$
V=\frac{1}{2} \sum_{i=1}^{n-1} \sum_{j=i+1}^{n} k_{i, j}\left\{\left\|x_{i}(0)-x_{j}(0)\right\|-\left\|\delta_{i}(t)-\delta_{j}(t)\right\|\right\}^{2},
$$

where $k_{i, j}$ is the spring constant between the $i$ th and $j$ th atoms. By using a Taylor series approximation, we can rewrite Equation (3) as

$$
V=\frac{1}{2} \sum_{i=1}^{n-1} \sum_{j=i+1}^{n} k_{i, j}\left(\delta_{i}(t)-\delta_{j}(t)\right)^{T}\left(\frac{\left(x_{i}(0)-x_{j}(0)\right)\left(x_{i}(0)-x_{j}(0)\right)^{T}}{\left\|x_{i}(0)-x_{j}(0)\right\|}\right)\left(\delta_{i}(t)-\delta_{j}(t)\right) .
$$

Then, we can derive the EOM using Lagrange's equation.

$$
\frac{d}{d t}\left(\frac{\partial L}{\partial \dot{\delta}_{i}}\right)-\frac{\partial L}{\partial \delta_{i}}=0
$$


Here, $\mathrm{L}=T-V$. Therefore, the EOM for the system is

$$
M \ddot{\delta}+K \delta=0 .
$$

Next, we also performed SCENM-based NMA to analyze vibrational characteristics of the DNA $3 \mathrm{D}$ buckyball structure. Suppose that there is a system of $q$ repeated subunits. We can then apply the symmetric boundary conditions to the system, such as intra-connectivity from a single subunit and the inter-connectivity from adjacent subunits. Because of the number of subunits $q$, we can rewrite $\vec{\delta}$ by dividing into $q$ parts as

$$
\vec{\delta}=\left[\vec{\Delta}_{1}^{\mathrm{T}}, \cdots, \vec{\Delta}_{q}^{\mathrm{T}}\right]^{\mathrm{T}}
$$

where $\Delta_{i}^{\mathrm{T}}=\left[\vec{\delta}_{1}^{\mathrm{T}}, \cdots, \vec{\delta}_{m}^{\mathrm{T}}\right]$ is the $x, y, z$ coordinates of a single subunit which has $m$ atoms $(i=1,2, \ldots, q)$. If there are $q$ rotation matrices for this structure, we can rewrite the coordinates of each subunit by using the first subunit and rotation matrices.

$$
\Delta_{i}=R_{i} \Delta_{1}
$$

Here, $R_{i}=\left[\begin{array}{ccc}R_{1}^{\prime} & \cdots & 0 \\ \vdots & \ddots & \vdots \\ 0 & \cdots & R^{\prime}{ }_{m}\end{array}\right]$ is a rotation matrix between the first subunit and $i$ th subunit and is a $3 m$ by $3 m$ matrix.

Then, we can construct the reduced stiffness matrix $K_{\text {reduced }}$ for a single subunit by including the connectivity information as

$$
K_{\text {reduced }}=K_{1,1}+K_{1,2} R_{2}+\cdots+K_{1, q} R_{q}
$$

where $R_{2}$ and $R_{q}$ are rotation matrices, and $K_{1,1}, K_{1,2}$, and $K_{1, q}$ are the inter-connections between subunits. More details are described in Supplementary Material. Therefore, we can rewrite the EOM of the system to use $M_{\text {reduced }}$, which is an inertia matrix of a single subunit and $K_{\text {reduced }}$.

$$
M_{\text {reduced }} \stackrel{\vec{\delta}}{\delta}+K_{\text {reduced }} \vec{\delta}=0
$$

Solving Equation (6) from MWCENM yields the vibrational motion and its frequency data. We can also obtain both vibrational motion and its frequency data of a single subunit by solving Equation (10) from SCENM. Vibrational motion of the whole structure can be recomposed to duplicate as a number of subunit copies. Otherwise, solving the procedure for the EOM is the same.

Supplementary Materials: Supplementary materials can be found at http:/ / www.mdpi.com/1422-0067/19/7/ $1895 /$ s1.

Author Contributions: S.J. and B.H.L. constructed the computer models and performed simulations. S.K., B.K., and A.T. conducted the experiments. M.K.K. and S.H.P. coordinated and supervised simulations and experiments, respectively. All authors wrote and reviewed the manuscript together, and S.J. and B.K. prepared the figures.

Funding: This research was supported by the Basic Science Research Program through the National Research Foundation of Korea (NRF), funded by the Ministry of Education (2015R1D1A1A01057280, M.K.K.), (2018R1A2B6008094, S.H.P.).

Conflicts of Interest: The authors declare no conflict of interest. 


\section{References}

1. Nummelin, S.; Kommeri, J.; Kostiainen, M.A.; Linko, V. Evolution of structural DNA nanotechnology. Adv. Mater. 2018, 30, 1703721. [CrossRef] [PubMed]

2. Wang, P.; Meyer, T.A.; Pan, V.; Dutta, P.K.; Ke, Y. The beauty and utility of DNA origami. Chem 2017, 2, 359-382. [CrossRef]

3. Bathe, M.; Rothemund, P.W.K. DNA nanotechnology: A foundation for programmable nanoscale materials. MRS Bull. 2017, 42, 882-888. [CrossRef]

4. Yin, P.; Hariadi, R.F.; Sahu, S.; Choi, H.M.; Park, S.H.; LaBean, T.H.; Reif, J.H. Programming DNA tube circumferences. Science 2008, 321, 824-826. [CrossRef] [PubMed]

5. Winfree, E.; Liu, F.; Wenzler, L.A.; Seeman, N.C. Design and self-assembly of two-dimensional DNA crystals. Nature 1998, 394, 539-544. [CrossRef] [PubMed]

6. Liu, D.; Wang, M.; Deng, Z.; Walulu, R.; Mao, C. Tensegrity: Construction of rigid DNA triangle with flexible four-arm DNA junctions. J. Am. Chem. Soc. 2004, 126, 2324-2325. [CrossRef] [PubMed]

7. Hamada, S.; Murata, S. Substrate-assisted assembly of interconnected single-duplex DNA nanostructures. Angew. Chem. Int. Ed. Engl. 2009, 48, 6820-6823. [CrossRef] [PubMed]

8. Zheng, J.; Birktoft, J.J.; Chen, Y.; Wang, T.; Sha, R.; Constantinou, P.E.; Ginell, S.L.; Mao, C.; Seeman, N.C. From molecular to macroscopic via the rational design of a self-assembled 3D DNA crystal. Nature 2009, 461, 74-77. [CrossRef] [PubMed]

9. Rothemund, P.W.K. Folding DNA to create nanoscale shapes and patterns. Nature 2006, 440, $297-302$. [CrossRef] [PubMed]

10. Wei, B.; Dai, M.; Yin, P. Complex shapes self-assembled from single-stranded DNA tiles. Nature 2012, 485, 623-626. [CrossRef] [PubMed]

11. Ke, Y.; Ong, L.L.; Shih, W.M.; Yin, P. Three-dimensional structures self-assembled from DNA bricks. Science 2012, 338, 1177-1183. [CrossRef] [PubMed]

12. Lee, J.; Hamada, S.; Amin, R.; Kim, S.; Kulkarni, A.; Kim, T.; Roh, Y.; Murata, S.; Park, S.H. Size-controllable DNA rings with copper-ion modification. Small 2012, 8, 374-377. [CrossRef] [PubMed]

13. Kim, J.; Lee, J.; Hamada, S.; Murata, S.; Park, S.H. Self-replication of DNA rings. Nat. Nanotechnol. 2015, 10, 528-533. [CrossRef] [PubMed]

14. Chen, J.; Seeman, N.C. Synthesis from DNA of a molecule with the connectivity of a cube. Nature 1991, 350, 631-633. [CrossRef] [PubMed]

15. Shih, W.M.; Quispe, J.D.; Joyce, G.F. A 1.7-kilobase single-stranded DNA that folds into a nanoscale octahedron. Nature 2004, 427, 618-621. [CrossRef] [PubMed]

16. He, Y.; Ye, T.; Su, M.; Zhang, C.; Ribbe, A.E.; Jiang, W.; Mao, C. Hierarchical self-assembly of DNA into symmetric supramolecular polyhedra. Nature 2008, 452, 198-201. [CrossRef] [PubMed]

17. Douglas, S.M.; Dietz, H.; Liedl, T.; Hogberg, B.; Graf, F.; Shih, W.M. Self-assembly of DNA into nanoscale three-dimensional shapes. Nature 2009, 459, 414-418. [CrossRef] [PubMed]

18. Bahar, I.; Rader, A.J. Coarse-grained normal mode analysis in structural biology. Curr. Opin. Struct. Biol. 2005, 15, 586-592. [CrossRef] [PubMed]

19. Kim, M.H.; Seo, S.; Jeong, J.I.; Kim, B.J.; Liu, W.K.; Lim, B.S.; Choi, J.B.; Kim, M.K. A mass weighted chemical elastic network model elucidates closed form domain motions in proteins. Protein Sci. 2013, 22, 605-613. [CrossRef] [PubMed]

20. Kim, B.; Jo, S.; Son, J.; Kim, J.; Kim, M.H.; Hwang, S.U.; Dugasani, S.R.; Kim, B.D.; Liu, W.K.; Kim, M.K.; et al. Ternary and senary representations using DNA double-crossover tiles. Nanotechnology 2014, 25, 105601. [CrossRef] [PubMed]

21. Jo, S.; Son, J.; Lee, B.H.; Dugasani, S.R.; Park, S.H.; Kim, M.K. Vibrational characteristics of DNA nanostructures obtained through a mass-weighted chemical elastic network model. RSC Adv. 2017, 7, 47190-47195. [CrossRef]

22. Isami, S.; Sakamoto, N.; Nishimori, H.; Awazu, A. Simple elastic network models for exhaustive analysis of long double-stranded DNA dynamics with sequence geometry dependence. PLoS ONE 2015, 10, e0143760. [CrossRef] [PubMed] 
23. Gonzalez, A.L.; Teixidó, J.; Borrell, J.I.; Estrada-Tejedor, R. On the applicability of elastic network models for the study of RNA CUG trinucleotide repeat overexpansion. PLoS ONE 2016, 11, e0152049. [CrossRef] [PubMed]

24. Pinamonti, G.; Bottaro, S.; Micheletti, C.; Bussi, G. Elastic network models for RNA: A comparative assessment with molecular dynamics and SHAPE experiments. Nucleic Acids Res. 2015, 43, 7260-7269. [CrossRef] [PubMed]

25. Zimmermann, M.T.; Jernigan, R.L. Elastic network models capture the motions apparent within ensembles of RNA structures. RNA 2014, 20, 792-804. [CrossRef] [PubMed]

26. Setny, P.; Zacharias, M. Elastic network models of nucleic acids flexibility. J. Chem. Theory Comput. 2013, 9, 5460-5470. [CrossRef] [PubMed]

27. Qian, P.F.; Seo, S.; Kim, J.; Kim, S.; Lim, B.S.; Liu, W.K.; Kim, B.J.; LaBean, T.H.; Park, S.H.; Kim, M.K. DNA nanotube formation based on normal mode analysis. Nanotechnology 2012, 23, 105704. [CrossRef] [PubMed]

28. Son, J.; Jo, S.; Song, Y.; Lee, B.H.; Kim, M.K.; Kim, B.D.; Park, S.H. Construction of foldback intercoil DNA nanostructures and analysis of their vibrational modes. J. Phys. Chem. C 2018, 122, 2890-2896. [CrossRef]

29. Hu, G.; He, L.; Iacovelli, F.; Falconi, M. Intrinsic dynamics analysis of a DNA octahedron by elastic network model. Molecules 2017, 22, 145. [CrossRef] [PubMed]

30. Afonin, K.A.; Kasprzak, W.; Bindewald, E.; Puppala, P.S.; Diehl, A.R.; Hall, K.T.; Kim, T.J.; Zimmermann, M.T.; Jernigan, R.L.; Jaeger, L.; et al. Computational and experimental characterization of RNA cubic nanoscaffolds. Methods 2014, 67, 256-265. [CrossRef] [PubMed]

31. Alves, C.; Iacovelli, F.; Falconi, M.; Cardamone, F.; Morozzo della Rocca, B.; de Oliveira, C.L.P.; Desideri, A. A simple and fast semiautomatic procedure for the atomistic modeling of complex DNA polyhedra. J. Chem. Inf. Model. 2016, 56, 941-949. [CrossRef] [PubMed]

32. Lee, H.; Seo, S.; Kim, M.H.; Choi, J.B.; Kim, S.M.; Jeon, T.J.; Kim, M.K. Opening and closing of a toroidal group II chaperonin revealed by a symmetry constrained elastic network model. Protein Sci. 2014, 23, 703-713. [CrossRef] [PubMed]

33. Conway, J.F.; Wikoff, W.R.; Cheng, N.; Duda, R.L.; Hendrix, R.W.; Johnson, J.E.; Steven, A.C. Virus maturation involving large subunit rotations and local refolding. Science 2001, 292, 744-748. [CrossRef] [PubMed]

34. Schuyler, A.D.; Chirikjian, G.S. Normal mode analysis of proteins: A comparison of rigid cluster modes with $\mathrm{C}_{\alpha}$ coarse graining. J. Mol. Graph. Model. 2004, 22, 183-193. [CrossRef]

35. Tozzini, V. Coarse-grained models for proteins. Curr. Opin. Cell Biol. 2005, 15, 144-150. [CrossRef] [PubMed]

36. Doruker, P.; Atilgan, A.R.; Bahar, I. Dynamics of proteins predicted by molecular dynamics simulations and analytical approaches: Application to alpha-amylase inhibitor. Proteins 2000, 40, 512-524. [CrossRef]

37. Atilgan, A.R.; Durell, S.R.; Jernigan, R.L.; Demirel, M.C.; Keskin, O.; Bahar, I. Anisotropy of fluctuation dynamics of proteins with an elastic network model. Biophys. J. 2001, 80, 505-515. [CrossRef]

38. Hinsen, K. Analysis of domain motions by approximate normal mode calculations. Proteins 1998, 33, 417-429. [CrossRef]

39. Kim, M.K.; Jernigan, R.L.; Chirikjian, G.S. An elastic network model of HK97 capsid maturation. J. Struct. Biol. 2003, 143, 107-117. [CrossRef]

40. Lee, B.H.; Jo, S.; Choi, M.; Kim, M.H.; Choi, J.B.; Kim, M.K. Normal mode analysis of Zika virus. Comput. Biol. Chem. 2018, 72, 53-61. [CrossRef] [PubMed]

41. Kim, M.H.; Lee, B.H.; Kim, M.K. Robust elastic network model: A general modeling for precise understanding of protein dynamics. J. Struct. Biol. 2015, 190, 338-347. [CrossRef] [PubMed]

(C) 2018 by the authors. Licensee MDPI, Basel, Switzerland. This article is an open access article distributed under the terms and conditions of the Creative Commons Attribution (CC BY) license (http://creativecommons.org/licenses/by/4.0/). 